\title{
Study on the Size Reduction Characteristics of Miscanthus sacchariflorus via Image Processing ${ }^{1}$
}

\author{
Hyoung-Woo Lee $\mathbb{D}^{2, \dagger} \cdot$ Jae-Won Lee ${ }^{2} \cdot$ Sung-Ho Gong ${ }^{3} \cdot$ Yeon-Sang Song ${ }^{4}$
}

\begin{abstract}
Size reduction is an important pre-processing operation for utilizing biomass as a sustainable resource in industrial-scale energy production and as a raw material for other industries. This work investigates the size reduction characteristics of air-dried Miscanthus sacchariflorus Goedae-Uksae 1 (Amur silver grass) via image processing and identifies the morphological characteristics of comminuted and screened $M$. sacchariflorus. At chopping lengths of 18, 40, 80, and $160 \mathrm{~mm}, 81 \%, 77 \%, 78 \%$, and $76 \%$ of the particles, respectively, passed through a $4-\mathrm{mm}$ sieve. Even a knife mill with a very small screen aperture $(>1 \mathrm{~mm})$ admitted over $10 \%$ of the particles. The average circularity and aspect ratio of the particles were $<0.30$ and $>10$, respectively. These results confirm that in all preparation modes, most $M$. sacchariflorus particles were needle-like in shape, irrespective of the type of preparation.
\end{abstract}

Keywords: size reduction, biomass, particle size distribution, image processing, Miscanthus sacchariflorus Goedae-Uksae 1

\section{INTRODUCTION}

Size reduction is a vital pre-processing operation associated with the use of biomass in energy production and as a resource for other industries. Correct design and implementation of the size reduction process along with the necessary equipment enhances the efficiency of the biomass and raw material preparation (Naimi et al., 2006). Moreover, the quality of the manufactured biomass pellets depends on the size, shape, and uniformity of the raw material. Lehmann et al. (2012) reported that shearing comminution of Miscanthus sacchariflorus (Amur silver grass) yields a highly fibrous material that compacts more readily in the press channels than normal shavings. In the biofuel (e.g., bio-ethanol) industry, reducing the biomass to appropriately sized particles improves the efficiency of biofuel conversion (Zhang, 2014). Hwang et al. (2012) investigated the effect of particle size of woody biomass on the features of pyrolytic products.

For simple, low-cost extraction, biomass components with different physical properties can be physically separated by devices such as vibrating sieves, air-screen cleaners, and classifiers. Sieves are used for large-scale

${ }^{1}$ Date Received March 7, 2018, Date Accepted June 19, 2018

2 College of Agriculture and Life Science, Chonnam National University, Gwangju 61186, Republic of Korea

${ }^{3}$ Intertek Kimsco Co., Ltd., 34 Yongam-gil, Cheongnyang-myeon, Ulju-gun, Ulsan 44989, Republic of Korea

${ }^{4}$ Bioenergy Crop Research Center, National Institute of Crop Science, Rural Development Administration, 199 Muan-ro, 58545, Republic of Korea

† Corresponding author: Hyoung-Woo Lee (e-mail: dryingeng@daum.net, ORCID: 0000-0001-6451-325X) 
particle separation in industries and for particle size distribution analysis in laboratories. Industrial applications of sieves include the separation of coal particles and the purification of pharmaceutical materials. However, most particle separation research has focused on the dimensions of the sieve aperture without considering the sizes of the particles retained on the sieve. Conventional research assumes spherical particles with similar dimensions in orthogonal directions. The behavior of uniformly shaped particles passing through an aperture has received considerable attention (Yang, 2007), whereas that of heterogeneous irregular particles, e.g., biomass, has been researched less.

Although engineering has addressed these challenges, the particle size reduction of biomass remains poorly understood. In particular, the effect of the size reduction process on the size distribution and shape of the refined particles has been insufficiently researched. This has hampered the selection of the size reduction process and the development of a classification method that minimizes the energy consumption of size reduction and maximizes the productivity of products manufactured from biomass.

The sizes of biomass particles with needle-like shapes, such as those of M. sacchariflorus, cannot be determined using standard sieving methods. Instead, as recommended by Chaloupkova et al. (2016), they can be determined using machine vision and image analysis techniques.

The present work elucidates the size reduction characteristics of air-dried M. sacchariflorus GoedaeUksae 1 via image processing to elucidate the importance of optimizing the size reduction process.

\section{MATERIALS and METHODS}

\subsection{Material preparation}

Air-dried samples of M. sacchariflorus Goedae-Uksae
1 were provided by the Bioenergy Crop Research Center, Muan, Korea. The average moisture content of the samples (measured using the oven-drying method) was $8 \%$ (wet basis). The samples were chopped into four lengths (18, 40, 80 and $160 \mathrm{~mm}$ ) using a forage chopper (Model No. SC4500, Hwangso Co., Korea) operated by a $2.25-\mathrm{kW}$ electric motor. The chopping length was varied by altering the rotational speed of the motor.

The chopped samples of different lengths were comminuted in a laboratory-scale knife mill (Model No. LKM2015, Drying Engineering, Inc., Korea) equipped with a $3.75-\mathrm{kW}$ electric motor operated at $1720 \mathrm{rpm}$. Four knives (length: $107 \mathrm{~mm}$ and width: $30 \mathrm{~mm}$ ) were mounted on the rotor inside the milling chamber, and two cutting bars were mounted on the inside wall of the milling chamber. Biomass was placed between the knives and the cutting bars and split into particles. The knives and cutting bars were separated by a 3-mm gap. The varied screen sizes used herein were $3,5,7$, and $9 \mathrm{~mm}$. Particles smaller than the screen aperture were passed during knife milling, whereas larger particles were re-circulated and re-milled.

\subsection{Size distribution analysis}

The biomass particles produced via knife milling are necessarily inhomogeneous. The particle size distributions were determined using a 5-tier sieve shaker (with sieve sizes of 1.00, 2.00, 4.00, 6.35, and 9.50 $\mathrm{mm})$. A $100 \mathrm{~g}$ sample of the comminuted $M$. sacchariflorus particles was placed on the top sieve and shaken for $5 \mathrm{~min}$. The particles retained on each sieve after shaking were collected and weighed. The particle weights in the five size ranges $(>9.50 \mathrm{~mm}$, 6.35-9.50 mm, 4.00-6.35 mm, 2.00-4.00 mm, 1.00-2.00 $\mathrm{mm}$, and $<1.00 \mathrm{~mm}$ ) were translated to particle size distribution data. The reported data are the average data of triplicate analyses. 


\subsection{Particle shape analysis}

A flatbed scanner (Cano-scan 4400F, Canon U.S.A. Inc., Lake Success, NY) provided digital images (4800 $\times 9600$ dpi) that were used to analyze the particle shapes. For imaging, $1 \mathrm{~g}$ of each sample was spread onto a transparent sheet and placed on the scanner glass. After spreading, the particles were completely separated with no contact or overlap. A sheet of black paper was installed as the background to optimize the contrast during the image acquisition.

The acquired images were converted to 8-bit grayscale images for binarization and then analyzed using the ImageJ software package. ImageJ is a public-domain, Java-based image processing program developed at the National Institutes of Health, USA (Ferreira and Rasband, 2012).

The apparent size of the particles is affected by the threshold level of the binary conversion. For instance, a low-level threshold level (0-255) generates more white areas around the area of the projected particle because the black background is whitened and a gray transition area appears between the particles. Therefore, the sizes of the particles determined using ImageJ were compared with those measured using a caliper. Finally, the 8-bit grayscale images were converted to binary images with a threshold of 100 . Any particles sized below $30 \mu \mathrm{m} 2$ were removed via filtering.

The areas, perimeters, circularities, and aspect ratios of the particles on the images were determined. The areas and perimeters were expressed in pixels. The circularity value was 1.0 for completely round particles and decreased with increasing elongation of the particles. The circularities and aspect ratios were respectively calculated as follows:

$$
\text { Circularity }=4 \pi \times\left(\frac{\text { Particle area }}{\text { Particle perimeter }}\right)^{2} \text {, }
$$

$$
\text { Aspect ratio }=\frac{\text { Major axis of the particle }(\text { fitted to an ellipse })}{\text { Minor axis of the particle }(\text { fitted to an ellipse })}
$$

The reported results are the average values of six determinations.

\section{RESULTS and DISCUSSION}

\subsection{Particle size distribution}

Figs. 2-5 show the effects of chopping length and screen size of the knife mill on the size distribution of $M$. sacchariflorus particles. The size distributions are similar in each figure, indicating that the chopping

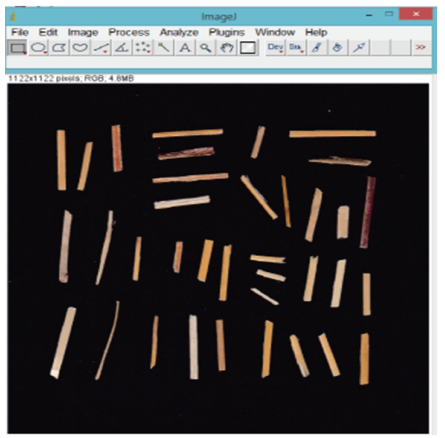

(a)

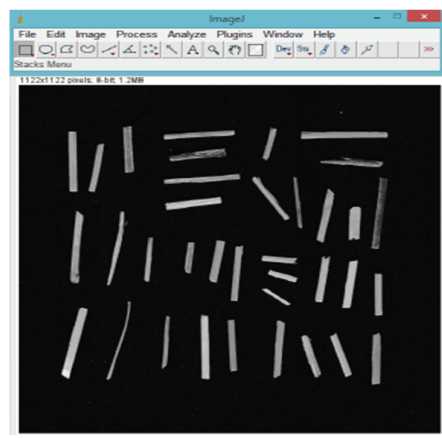

(b)

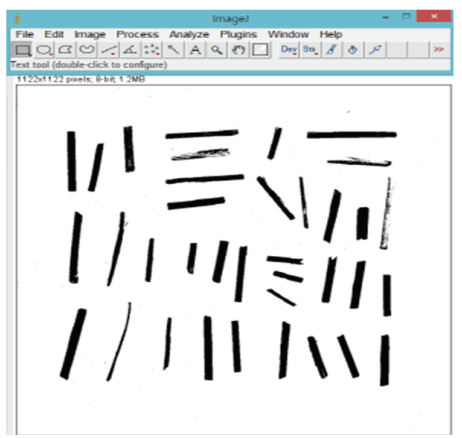

(c)

Fig. 1. Scanned image (a), 8-bit grayscale image (b), and binary image (c) of comminuted and screened Miscanthus sacchariflorus Goedae-Uksae 1. 


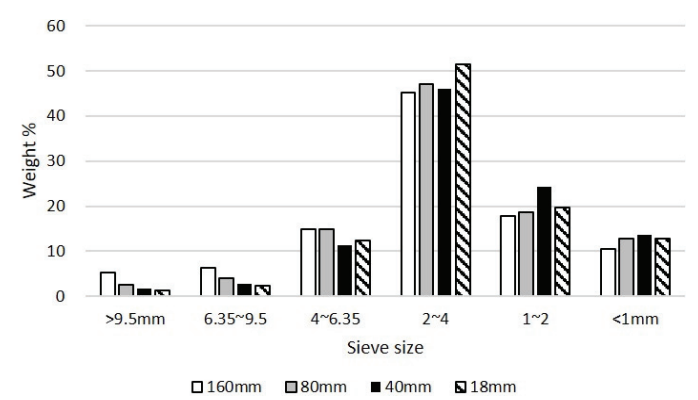

Fig. 2 Size distribution of $M$. sacchariflorus GoedaeUksae 1 particles comminuted using a knife mill with different chopping lengths (18, 40, 80, and $160 \mathrm{~mm}$ ) and a screen aperture size of $9 \mathrm{~mm}$.

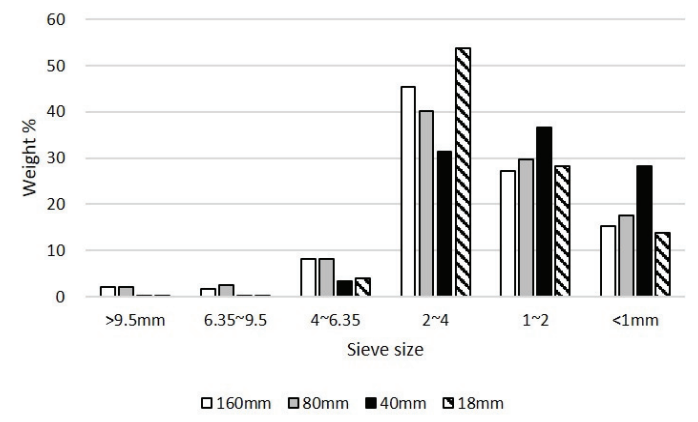

Fig. 3. Size distribution of $M$. sacchariflorus GoedaeUksae 1 comminuted using a knife mill with different chopping lengths (18, 40, 80, and $160 \mathrm{~mm}$ ) and a screen aperture size of $7 \mathrm{~mm}$.

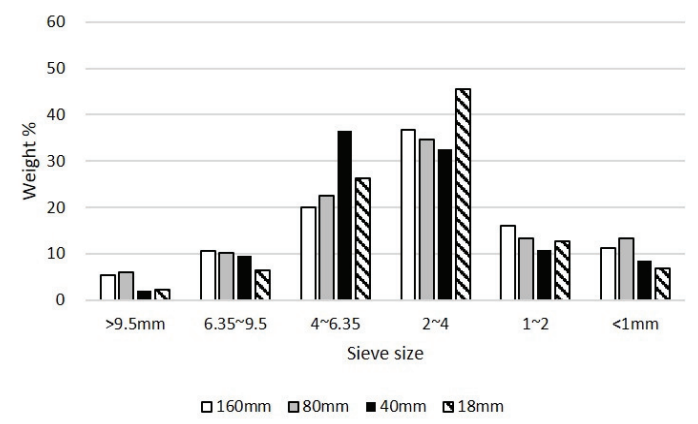

Fig. 4. Size distribution of $M$. sacchariflorus GoedaeUksae 1 particles comminuted using a knife mill with different chopping lengths (18, 40, 80, and $160 \mathrm{~mm}$ ) and a screen aperture size of $5 \mathrm{~mm}$.

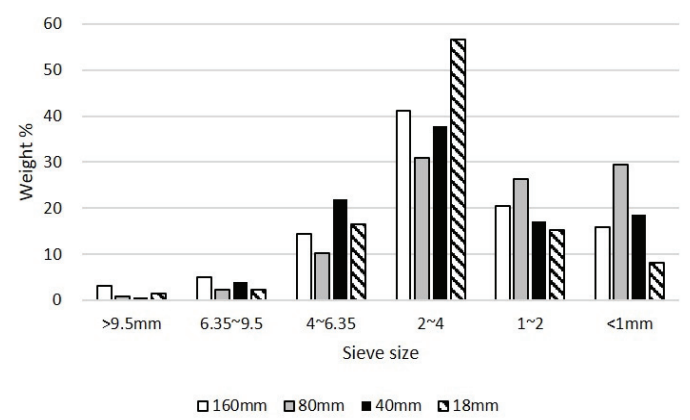

Fig. 5. Size distribution of $M$. sacchariflorus GoedaeUksae 1 particles comminuted using a knife mill with different chopping lengths $(18,40,80$, and $160 \mathrm{~mm}$ ) and a screen aperture size of $3 \mathrm{~mm}$.

length and screen size of the knife mill did not significantly affect the results. At chopping lengths of $18,40,80$, and $160 \mathrm{~mm}, 81 \%, 77 \%, 78 \%$, and $76 \%$ of the particles, respectively, passed through a $4-\mathrm{mm}$ sieve. Lee (2016) reported that the screen size of the knife mill seriously affects the size distribution of wooden particles. In contrast, Chaloupkova et al. (2016) concluded that chopped and comminuted $M$. sacchariflorus particles are needle-like in shape and can easily pass through the screen aperture, regardless of their length.

Even the smallest screen aperture of the knife mill $(<1 \mathrm{~mm})$ admitted $10 \%$ of the particles, indicating a significant degree of comminution in the knife mill chamber. The high crushing phenomenon is attributed to the pith in $M$. sacchariflorus, which is easily pulverized into small particles.

\subsection{Particle shape}

The average areas (in square pixels) of the $M$. sacchariflorus particles comminuted using a knife mill under different conditions are listed in Table 1. Consistent with expectations, the average area decreased with decreasing chopping length. There was no significant relationship between the area of the particles 
Table 1. Average areas (in square pixels) of comminuted Miscanthus sacchariflorus particles passed through knife mills with different chopping lengths and screen aperture sizes.

\begin{tabular}{ccccc}
\hline Chopping & \multicolumn{4}{c}{ Screen Aperture Size of the Knife Mill } \\
\cline { 2 - 5 } Length & $3 \mathrm{~mm}$ & $5 \mathrm{~mm}$ & $7 \mathrm{~mm}$ & $9 \mathrm{~mm}$ \\
\hline \hline $18 \mathrm{~mm}$ & 8,130 & 7,330 & 5,382 & 9,544 \\
$40 \mathrm{~mm}$ & 9,270 & 10,546 & 8,704 & 11,068 \\
$80 \mathrm{~mm}$ & 10,958 & 12,377 & 10,260 & 11,575 \\
$160 \mathrm{~mm}$ & 9,637 & 12,002 & 10,463 & 11,796 \\
\hline
\end{tabular}

and the screen aperture size of the knife mill. Some narrow and long particles with relatively large areas failed to pass through a given screen aperture, while others that were insufficiently comminuted or not comminuted at all passed through the given screen aperture. This demonstrates that the particle size distribution of $M$. sacchariflorus cannot be accurately analyzed using the vibrating sieve method.

The average circularities of the $M$. sacchariflorus particles are shown in Fig. 6. Particles chopped to 18 $\mathrm{mm}$ before comminution using the knife mill were more circular than those chopped to other lengths. However, the average circularities at all chopping lengths were lower than 0.3. There were no significant differences among the data at chopping lengths $>18 \mathrm{~mm}$.

Fig. 7 shows the effects of chopping lengths and screen aperture sizes of the knife mill on the average aspect ratios of the comminuted $M$. sacchariflorus particles. Most aspect ratios exceeded 10, indicating that the particles were over 10 times longer than their width. At chopping lengths of 40 and $80 \mathrm{~mm}$, the aspect ratios decreased as the screen aperture size increased. No trends were apparent at chopping lengths of 18 and $160 \mathrm{~mm}$.

The low average circularity and high average aspect ratios of the chopped and comminuted $M$. sacchariflorus samples imply a dominance of needle-like particles in all preparation modes. In applications requiring a strict particle size of the raw material, e.g., the manufacture of biomass pellets, the vibrating sieve method appears to be unsuitable. Certainly, it failed to separate appropriately sized particles from comminuted $M$. sacchariflorus. Womac et al. (2007) recommended a multiple-stage grinding process for proper size reduction of biomass.

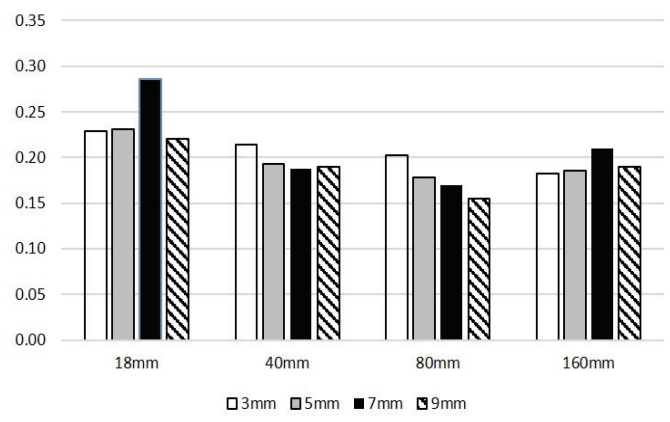

Fig. 6. Average circularities of $M$. sacchariflorus Goedae-Uksae 1 particles comminuted using a knife mill with different chopping lengths $(18,40,80$, and $160 \mathrm{~mm}$ ) and screen aperture sizes (3, 5, 7, and $9 \mathrm{~mm}$ ).

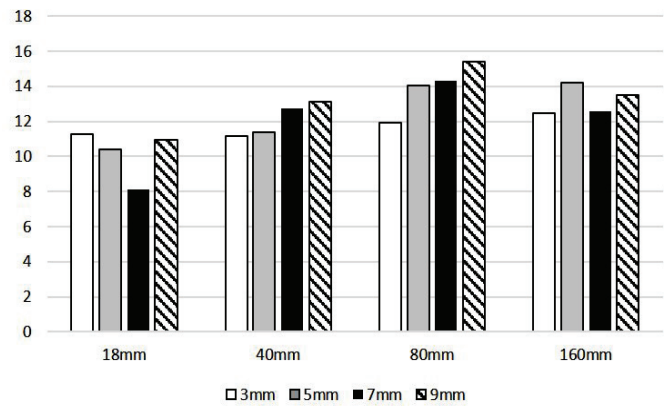

Fig. 7. Average aspect ratios of M. sacchariflorus Goedae-Uksae 1 particles comminuted using a knife mill with different chopping lengths $(18,40,80$, and $160 \mathrm{~mm}$ ) and screen aperture sizes (3, 5, 7, and $9 \mathrm{~mm}$ ). 


\section{CONCLUSION}

The size distributions of comminuted M. sacchariflorus Goedae-Uksae 1 particles were investigated using different chopping lengths and screen aperture sizes of a knife mill. In addition, the particle shapes were investigated using image processing techniques. The lengths of the chopped particles and the screen aperture sizes of the knife mill exerted no significant influence on the particle size distribution. The proportions of particles with chopped lengths of $18,40,80$, and 160 $\mathrm{mm}$ passing through a $4-\mathrm{mm}$ sieve were $81 \%, 77 \%$, $78 \%$, and $76 \%$, respectively. The low average circularity and high average aspect ratios indicated a dominance of needle-like particles in the prepared samples, regardless of the preparation mode. Furthermore, the vibrating sieve method appears to be unsuitable for separating appropriately sized particles from comminuted M. sacchariflorus.

\section{ACKNOWLEDGMENT}

This research was supported by the National Institute of Crop Science, Rural Development Administration, Korea (Grant No. PJ012577052017).

\section{REFERENCES}

Chaloupkova, V., Ivanova, T., Havrland, B. 2016. Sieve analysis of biomass: Accurate method for determination of particle size distribution. Engineering for Rural Development, Czech University of Life Sciences Prague 25: 1012-1017. Ferreira, T., Rasband, W. 2012. ImageJ user guide book.
National Institutes of Health, USA.

Hwang, H.W., Oh, S.Y., Kim, J.Y., Lee, S.M., Cho, T.S., Choi, J.W. 2012. Effect of particle size and moisture content of woody biomass on the feature of pyrolytic products. Journal of the Korean Wood Science and Technology 40(6): 445-453.

Lee, H.W. 2016. Size reduction characteristics of yellow poplar in a laboratory knife mill. Journal of the Korean Wood Science and Technology 44(2): 166-171.

Lehmann, B., Schroder, H.W., Wollenberg, R., Repke, J.R. 2012. Effect of miscanthus addition and different grinding processes on the quality of wood pellets. Biomass and Bioenergy 44: 150 159.

Naimi, L.J., Sokhansanj, S., Mani, S., Hoque, M., Bi. T. 2006. Cost and performance of woody biomass size reduction for energy production. Proceedings of CSBE/SCGAB 2006 Annual Conference, Edmonton, Alberta, Canada.

Womac, A.R., Igathinathane, C., Bitra, P., Miu, P., Yang, T., Sokhansanj, S., Narayan, S. 2007. Biomass pre-processing size reduction with instrumented mills. 2007 ASABE Annual International Meeting Presentation, Minneapolis, Minnesota, USA.

Yang, Y. 2007. Image and sieve analysis of biomass particle sizes and separation after size reduction. Master Theses, University of Tennessee, Knoxville, USA.

Zhang, M. 2014. Size reduction of cellulosic biomass for biofuel manufacturing. Ph.D. thesis, Department of Industrial \& Manufacturing Systems Engineering, College of Engineering, Kansas State University, USA. 